ACTA UNIVERSITATIS LODZIENSIS

Folia Litteraria Romanica 15, 2020

https://doi.org/10.18778/1505-9065.15.11

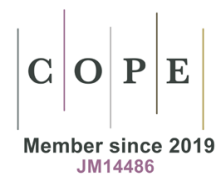

Tomasz Szymański

Université de Wrocław

(iD) ORCID ID : 0000-0002-2051-0003

tomasz.szymanski@uwr.edu.pl

\title{
« Aimer religieusement le monde et la vie » : la réponse de Pierre Leroux au contemptus mundi
}

\begin{abstract}
RÉSUMÉ
Dans son ouvrage De l'humanité, de son principe et de son avenir (1840), Pierre Leroux cherche à établir la "vraie définition de la religion ", en visant à une synthèse des traditions religieuses de l'humanité et de la philosophie moderne. Aucune des réponses philosophiques que l'homme a tenté jusqu'ici de donner à la question du bonheur et du souverain bien (stoïcisme, épicuréisme, platonisme, christianisme) ne peut être jugée comme satisfaisante. Entre matérialisme qui méprise l'esprit d'un côté et spiritualisme qui méprise la nature et le monde de l'autre, Leroux cherche une troisième voie : "Aimer religieusement le monde et la vie». Sa philosophie, qui est à la fois une manière de vivre et une réflexion herméneutique sur l'humanité, conduit à l'application véritable et socialement organisée du principe de l'amour, et dépassant égoïsme et altruisme dans la solidarité, permet de libérer la nature et la vie du mépris qui pesait sur elles pendant des siècles.
\end{abstract}

MOTS-CLÉS - Pierre Leroux, romantisme, contemptus mundi, religion, philosophie de la vie, philosophie comme manière de vivre, souverain bien

\footnotetext{
"To Love Religiously the World and the Life":

The Answer of Pierre Leroux to Contemptus Mundi
}

\begin{abstract}
SUMMARY
In his book De l'humanité, de son principe et de son avenir (1840), Pierre Leroux seeks to establish the "true definition of religion", aiming at a synthesis of the religious traditions of humanity and modern philosophy. None of the philosophical answers that man has hitherto attempted to give to the question of happiness and of the highest good (Stoicism, Epicureanism, Platonism, Christianity) can be judged as satisfying. Between materialism that despises the spirit on one side and spiritualism that despises nature and the world on the other, Leroux seeks a third way: "To love religiously the world and life". His philosophy, which is at the same time a way of life and a hermeneutic reflection on humanity, leads to real and socially organized application of the principle of love, and - transcending
\end{abstract}


in solidarity the opposition between selfishness and altruism - makes it possible to free nature and life from the contempt that weighed down on them for centuries.

KEYWORDS - Pierre Leroux, Romanticism, contemptus mundi, religion, philosophy of life, philosophy as a way of life, highest good

Le mépris du monde (le contemptus mundi), inséparable du thème de la uanitas, fait partie de l'héritage chrétien, et plus largement philosophique, de la culture occidentale. Il constitue l'un des pôles de l'attitude foncièrement ambivalente du christianisme par rapport au monde et à la nature créée - d'un côté bons en tant que créations divines, de l'autre - marqués et dégradés par le péché originel. D'un point de vue historique, cette « doctrine » du mépris du monde (c'est ainsi que la qualifie Robert Bultot, et à sa suite Juliusz Domański ${ }^{1}$ ) se développe de façon plus visible dans l'Antiquité tardive, surtout avec Augustin d'Hippone. Celui-ci disait que tout étant bon dans le monde créé, certains biens le sont davantage et d'autres moins, certains supérieurs à l'homme dans l'échelle des êtres - dont nous pouvons jouir (frui) - et d'autres inférieurs, dont nous devrions nous contenter d'user (uti). Jouir des biens inférieurs et user des biens supérieurs est l'apanage des mauvaises gens ${ }^{2}$.

Il est évident que cette conception s'inscrit dans une vision ontologique plus large, que le christianisme partageait alors avec la philosophie platonicienne, néoplatonicienne et pythagoricienne, qui valorise l'être spirituel et éternel au détriment de l'être matériel et passager. L'homme est fait à l'image et à la ressemblance de Dieu, certes, mais celui-ci étant esprit, c'est en l'âme que réside la vraie vie de l'homme. Le corps, même s'il n'est pas considéré ici, comme par exemple dans l'orphisme, comme prison de l'âme, est valorisé négativement et dans une large mesure méprisable. Il appartient en effet à cette nature déchue et corrompue, qui détourne l'homme de sa véritable destinée et de sa véritable patrie, surnaturelle et céleste. On n'était pas loin de constater que la nature de l'homme est angélique plutôt que proprement humaine - en effet, comme le constate Augustin en suivant les commentaires d'Ambrosiaster (pseudo-Ambroise) aux épîtres de Paul, l'homme a été créé pour remplacer dans le ciel les anges déchus en conséquence de leur révolte ${ }^{3}$. Toutes ces idées ne datent donc pas de l'Antiquité tardive, mais ont leurs racines dans la Bible, surtout dans le Nouveau Testament (écrits pauliniens et joanniques), que les Pères de l'Église commentent en essayant souvent de les associer avec la philosophie païenne, essentiellement spiritualiste. Le mépris du monde qui en résulte sera un leitmotiv de l'enseignement ecclésiastique durant tout le MoyenÂge et bien au-delà, trouvant son illustration exemplaire dans l'œuvre De miseria

1 J. Domański, Z dawnych rozważań o marności i pogardzie świata oraz nędzy i godności człowieka, Warszawa, Wydawnictwo IfiS PAN, 1997. Domański s'appuie notamment sur l'ouvrage en plusieurs tomes de R. Bultot, La Doctrine du mépris du monde; ibid., p. 15.

2 Ibid., p. 7-12.

3 Ibid., p. 15-20. 
condicionis humane siue de contemptu mundi (1195) par le moine Lothaire, futur pape Innocent III ${ }^{4}$.

C'est encore à cette tradition du mépris du monde que doit faire face Pierre Leroux, penseur, politique et philosophe du $\mathrm{XIX}^{\mathrm{e}}$ siècle ${ }^{5}$, auteur relativement méconnu malgré le rôle clef qu'il a joué dans le mouvement des idées à l'époque du romantisme, et l'influence qu'il a exercée sur toute une génération, notamment sur George Sand ou Ernest Renan. Leroux, né en 1797, est ouvrier typographe de métier. Il fonde Le Globe, revue d'abord libérale, puis saint-simonienne à partir de janvier 1831, Leroux s'étant enthousiasmé pour le mouvement de Saint-Simon pendant une année. Il sera aussi le rédacteur de la Revue encyclopédique, de l'Encyclopédie nouvelle (avec Jean Reynaud, 1834-1841) et de la Revue indépendante, avec George Sand, dont il devient le guide spirituel, et qui écrit de nombreux romans sous son influence. Elle n'est pas la seule à céder au charisme de Leroux, qui rassemble autour de soi à Boussac (1845) une communauté utopiste (qui regroupe jusqu'à quatrevingts membres), où on essaie de mettre en œuvre la théorie du circulus (consistant notamment à utiliser des excréments humains comme engrais). En 1848, Pierre Leroux est l'un des chefs de file des républicains (plus précisément des démocrates socialistes) et après le 2 décembre il se rend en exil à Londres, puis à Jersey, où il trouve Victor Hugo. Revenu en France après l'amnistie de 1859, il sombre toutefois progressivement dans la misère et meurt au temps de la Commune, en avril 1871. Paria, marginal et excentrique à bien des égards, loser pourrait-on dire aujourd'hui, Leroux est pourtant devenu pour certains une autorité, voire une icône, aussi bien politique et sociale que spirituelle et religieuse.

En effet, Leroux, «penseur de l'humanité » comme l'appelle Bruno Viard dans son livre ${ }^{6}$, est aussi penseur par excellence et paradoxalement religieux. Dans son ouvrage De l'humanité, de son principe et de son avenir $(1840)^{7}$, Leroux cherche à établir la « vraie définition de la religion ", en visant à une synthèse des différentes traditions religieuses de l'humanité et de la philosophie moderne à travers la conception d'une « Révélation éternelle et successive $»^{8}$. Dissident du saint-simonisme, Leroux prend pour point de départ et en même temps conteste

4 R. Bultot, "Mépris du monde, misère et dignité de l'homme, dans la pensée d'Innocent III », Cahiers de civilisation médiévale, octobre-décembre 1961, $4^{\mathrm{e}}$ année, $\mathrm{n}^{\circ}$ 16, p. 441-456, URL : https://www.persee.fr/doc/ccmed_0007-9731_1961_num_4_16_1205; consulté le 10.01.2019.

5 Au sujet de Leroux et de son œuvre, voir l'introduction de B. Viard à l'Anthologie de Pierre Leroux, inventeur du socialisme, établie et présentée par B. Viard, Lormont, Éditions Le Bord de l'eau, 2007, p. 7-65 ; B. Viard, Pierre Leroux, penseur de l'humanité, Cabris, Éditions Sulliver, 2009 ; P. Bénichou, Le Temps des prophètes. Doctrines de l'âge romantique, Paris, Gallimard, 1977, p. 330-358 ; D. O. Evans, Le Socialisme romantique : Pierre Leroux et ses contemporains, Paris, M. Rivière, 1948 ; S. Alexandrian, Le Socialisme romantique, Paris, Éditions du Seuil, 1979, p. 243-275.

6 Voir note 5 ci-dessus.

7 P. Leroux, De l'humanité, de son principe et de son avenir, t. 1, Paris, Perrotin, 1845 ; URL : http:// gallica.bnf.fr/ark:/12148/bpt6k454802b ; consulté le 10.01.2019.

8 Ibid., p. VII. 
la distinction en époques critiques et époques organiques admise par les saintsimoniens. Il ne s'agit pas de détruire purement et simplement l'ancien ordre social et religieux, comme l'on fait Voltaire, puis la Révolution. Il ne s'agit pas non plus de sombrer dans un nouveau dogmatisme ou dans une tyrannie exercée par le corps social sur l'individu. Leroux, qui cherche une troisième voie entre le socialisme absolu et l'individualisme absolu', tous deux également néfastes, semble adopter la même attitude vis-à-vis des questions religieuses et philosophiques : il ne s'agit pas de se couper du passé et de rejeter le christianisme ou la religion en bloc, ni de revenir à la religion traditionnelle instituée par l'Église - il faut chercher une troisième solution qui permettrait de concilier les exigences de la modernité avec les vérités cachées dans la tradition, autrement dit trouver un « axiome religieux » pour la pensée et la société, sans lequel celle-ci se décompose et court à sa perte : «C'est un axiome sur la vie, sur l'être, qui nous manque. C'est un axiome religieux. Que sommes-nous, qu'est chacun de nous en Dieu ? Quelle est la volonté du créateur eu nous donnant l'être à chaque moment de notre existence ? Où est notre vie, quel est l'objet de notre vie ? $\rangle^{10}$.

Ce sont donc les principales questions inscrites dans la condition humaine qui intéressent Leroux : les destinées de l'homme, conçu comme être « un » (être particulier dans lequel se reflète l'être collectif et universel, l'humanité), ainsi que la question du bonheur, à laquelle ont tenté de répondre tous les systèmes philosophiques, à commencer par ceux des anciens, qui ont en définitive pour but la pratique même de la vie. Disons tout de suite qu'aucune de ces réponses ne peut être jugée satisfaisante selon Leroux. Celui-ci pour simplifier en dénombre quatre, auxquelles se ramènent toutes les autres : le stoïcisme, l'épicuréisme, le platonisme et le christianisme. Ces quatre, quant à eux, se sont regroupés autour de deux courants majeurs adverses et également faux : le spiritualisme et le matérialisme ${ }^{11}$. Mais commençons par quelques observations de base : le bonheur absolu dans notre monde d'ici-bas (laissons pour l'instant de côté la question de l'au-delà) n'existe pas, il est une chimère, tous les philosophes s'en sont facilement aperçus. Nous courons après lui, mais il nous échappe, tout état de bien-être est éphémère et rien ne nous nous apaise réellement. Les religions ont toutes posé le diagnostic de la douleur universelle et du " gémissement de toute créature », pour reprendre la formule de l'épître aux Romains ${ }^{12}$ :

9 B. Viard, Pierre Leroux, op. cit., p. 23-32, 85-93 ; Anthologie, op. cit., p. 9-11. Leroux est l'auteur du néologisme " socialisme » (qu'il oppose à l'" individualisme », terme inventé par les saintsimoniens). Le socialisme a initialement pour lui un sens péjoratif, en tant que système social étouffant la liberté de l'individu. Plus tard, il lui attribue un sens républicain, qui témoigne de l'aspiration à une synthèse de la liberté, de l'égalité et de la fraternité.

${ }^{10}$ P. Leroux, De l'humanité, t. 1, op. cit., p. XIV.

11 Ibid., p. 7-8.

${ }^{12}$ Romains 8, 22-23. 
S. Paul, le grand poète, le grand théologien, a résumé d'un mot cette douleur universelle de la Nature quand il a dit Omnis creatura ingemiscit. Et la théologie chrétienne n'est pas la seule qui ait constaté ce gémissement de toute créature. / Toutes les antiques religions ont eu des mythes pour exprimer cette idée ; et nous venons de voir que les siècles dits de lumières et de philosophie, les siècles d'incrédulité, rendent également témoignage de la vanité de ce mot bonheur. Pourtant le mépris qu'on faisait du Ciel à ces époques aurait dû tourner au profit de la félicité terrestre. On voulait détrôner des religions vieillies, il fallait donc exalter la réalité aux dépens de leur idéal ; on n'avait que la terre, il fallait donc en jouir ; on ne croyait qu'au présent, il fallait donc en profiter [...] on a mis tout son génie à être égoïste avec art ; on a appelé cela sagesse, raison, philosophie : et, en fin de compte, on a été forcé d'avouer que le bonheur n'était pas fait pour l'homme ${ }^{13}$.

Le caractère illusoire du bonheur est le commencement de la sagesse, il peut donc mener à des attitudes différentes, voire opposées : les unes dictées par le mépris du monde et des vanités terrestres, les autres par le mépris du Ciel et de la religion. Car si le bonheur absolu n' existe pas, et s'il est impossible à atteindre sur cette terre, le malheur absolu n'existe pas lui non plus, et la douleur dans la vie est toujours équilibrée par le plaisir : «Or si S. Paul a dit que toute créature gémit, on pourrait dire avec autant de raison que toute créature sourit, et que le plaisir brille dans le monde comme la douleur $\rangle^{14}$. Par conséquent, on ne saurait rejeter la nature, se placer « contre le monde, contre la vie $»^{15}$, pour parler comme Michel Houellebecq, sous prétexte que la douleur y est présente et inévitable. Or, l'erreur cardinale des spiritualistes a consisté à inventer d'autres mondes (des « arrière-mondes », dirait Nietzsche $\left.{ }^{16}\right)$ pour pallier aux insuffisances de celui que nous habitons et compenser tous nos maux :

La terre, c'est-à-dire la vie telle que nous la connaissons, a été incomplètement appréciée ; et de là est venu et l'Éden chimérique et le Paradis chimérique. Les grands théologiens S. Paul et S. Augustin ont beau médire de la Nature, la Nature n'est pas aussi corrompue qu'ils le disent. La vie présente n'est pas uniquement dévouée au malheur. Aussi qu'est-il arrivé ? C'est que la Nature a toujours conservé ses partisans ; c'est que la vie présente s'est moquée de l'anathème jeté sur elle, et qu'on a fini depuis trois siècles par ne plus croire ni à l'Éden ni au Paradis ${ }^{17}$.

Toute la philosophie matérialiste du XVIII ${ }^{\mathrm{e}}$ siècle et sa critique de la religion, déiste ou athée, est une continuation de la pensée d'Épicure, ce « chasseur de spectres $»^{18}$, comme l'appelle Leroux après les Grecs. Épicure a réhabilité la nature et l'a acceptée telle qu'elle est. Sa pensée devait fleurir au moment du déclin des

${ }^{13}$ P. Leroux, De l'humanité, t. 1, op. cit., p. 15-16.

${ }^{14}$ Ibid., p. 21.

${ }^{15}$ M. Houellebecq, H. P. Lovecraft. Contre le monde, contre la vie, Monaco / Paris, Éditions du Rocher, 1991.

${ }^{16}$ F. Nietzsche, Ainsi parlait Zarathoustra, Paris, Éditions Gallimard, 1947. Dans la première partie de l'ouvrage, il est question des « hallucinés de l'arrière-monde».

${ }^{17}$ P. Leroux, De l'humanité, t. 1, op. cit., p. 19.

${ }^{18}$ Ibid., p. 42. 
grandes conceptions spiritualistes, qu'il s'agisse du paganisme ou du christianisme. En effet, le christianisme n'est aucunement responsable à lui seul du rejet de la vie qui est devenu le trait dominant de la philosophie occidentale. Constituant une reprise du platonisme, qui avait adopté une position modérée face au monde, la religion chrétienne a dû s'associer au stoïcisme pour développer pleinement sa doctrine du contemptus mundi :

\begin{abstract}
Mépriser complètement la vie, la laisser couler, comme ils disaient, en se réfugiant en soimême ; se regarder, relativement à cette vie, comme un spectateur ou tout au plus comme un acteur dans une comédie ; laisser au Destin la responsabilité de son œuvre ; ne pas songer à tempérer ses passions, mais les déraciner; se créer sans passions, faire de soi une intelligence libre, une liberté ; telle fut, comme chacun le sait, la morale des Stoïciens. Ils avaient pour cette vie un tel dédain, qu'ils s'attachèrent à démontrer que l'âme humaine était périssable, et que nous n'avions pas à craindre que la vie s'étendît au-delà de ce monde. Ils avaient pour ce monde un tel dégoût, qu'ils donnèrent à leur sage le droit de s'ôter la vie, comme une suite de sa liberté et une récompense de sa vertu ${ }^{19}$.
\end{abstract}

Que ce verdict de Leroux soit entièrement juste ou non, il est vrai que le mépris du monde n'est pas une invention chrétienne. Tout la tradition néoplatonicienne voit dans les ténèbres de la matière la sphère la plus éloignée de l'Un, dont il faut se libérer pour pouvoir le contempler et vivre de la vraie vie. Platon déjà, bien qu'il s'intéresse aux questions éthiques sous l'inspiration de Socrate, reprend dans sa métaphysique, via l'école de Pythagore, les systèmes orientaux considérant le corps comme une prison de l'âme. Rien de cela, comme nous le savons déjà, ne peut nous satisfaire selon Leroux. Certes, le platonisme, avec sa valorisation relative du monde, en tant que reflet du Beau, du Bien et du Vrai idéaux, peut passer pour une philosophie du perfectionnement successif plutôt que du salut instantané ${ }^{20}$ - en quoi il s'accorde avec la pensée de Leroux - mais il a fini par se faire supplanter par le christianisme. Or, celui-ci, en reprenant la métaphysique platonicienne, a adopté l'éthique stoïcienne de rejet du monde, en divinisant le Verbe dont parlait Platon, et en remplaçant la vertu par la grâce. Entre la figure du navigateur qui regarde le ciel pour s'orienter sur la mer, et celle de l'astronome qui contemple le ciel pour lui-même, l'Occident chrétien a opté pour cette seconde, quitte à fixer plus tard ses yeux exclusivement sur la terre, par réaction à un spiritualisme aliénant ${ }^{21}$. Quant à l'épicuréisme, on peut en distinguer deux genres : celui, originel d'Épicure, qui visait à rendre l'homme le moins malheureux possible ( « la nature du bien s'engendre de la fuite du mal »), et celui, antérieur, d'Aristippe, qui consiste en une recherche effrénée de la volupté. Or, c'est ce dernier, conduisant à un égoïsme déréglé, qui a toujours fini par prévaloir ${ }^{22}$.

\footnotetext{
${ }^{19}$ Ibid., p. 44-45.

${ }^{20}$ Ibid., p. 49-50.

${ }^{21}$ Ibid., p. 61-62.

${ }^{22} \mathrm{Ibid}$., p. 56-61. En réalité, Aristippe est fondateur de l'école cyrénaïque, et ne peut pas être considéré comme épicurien.
} 
Entre matérialisme qui méprise l'esprit d'un côté et spiritualisme qui méprise la nature et le monde de l'autre, entre une loi de l'attraction purement physique et une loi de l'attraction spirituelle, l'homme n'a pas à choisir. N'étant ni ange ni bête, étant lié au milieu humain et à l'humanité, il n'a qu'à rester fidèle à sa nature, qui est d'être « esprit-corps » ${ }^{23}$, ou « sensation - sentiment - connaissance», et ne se réduit pas à un seul de ces éléments ${ }^{24}$. De cette conception découle l'idée que Leroux se fait du souverain bien, partant de la devise « connais-toi toi-même ». Si pour Platon, celui-ci relève d'une réalité suprasensible, pour Épicure - de l'affirmation de la nature, pour Zénon - de la liberté intérieure, et pour les chrétiens - de l'amour révélé dans le Verbe incarné...

Aujourd'hui la Philosophie nous apprend que le souverain bien consiste à aimer religieusement le monde et la vie. Elle doit nous apprendre comment nous pouvons aimer religieusement le monde et la vie, comment, tout en restant dans la Nature et dans la vie, nous pouvons nous élever vers notre centre spirituel ${ }^{25}$.

Pour bien comprendre en quoi consiste la manière de vie philosophique que Leroux postule ici, il faut savoir ce qu'il entend par la vie :

Quant à la vie vivante, si je puis m'exprimer ainsi, nous pouvons bien nous en faire une idée, mais elle est indéfinissable. C'est la roue en mouvement, mais qu'est-ce que la roue en mouvement ? [...] Ainsi de la vie nous ne sommes jamais ni dans une idée ni dans un plaisir, ni dans une souffrance ; mais toujours nous sortons d'une idée, d'une jouissance ou d'une douleur, pour entrer dans une autre; nous ne sommes plus dans celle-là, nous ne sommes pas encore dans celle-ci, et déjà celle-ci est passée. / Le moment où je parle est déjà loin de moi $^{26}$. / Notre vie n'est donc pas même un point entre deux abîmes, comme dit Pascal, à moins d'entendre par ce point un point mathématique, un point sans dimension ${ }^{27}$.

Rythmée par l'" émersion d'un état antérieur et [1']immersion dans un état futur $\|^{28}$, la vie de l'homme est une aspiration incessante à la perfection ${ }^{29}$, au-delà de ses expériences ponctuelles et passagères ( $«$ je ne peins pas l'être, je peins

${ }^{23}$ Ibid., p. 81-95.

${ }^{24}$ Ibid., p. 99-111.

${ }^{25}$ Ibid., p. 94.

${ }^{26}$ Ce vers de Nicolas Boileau (Épître III) est pratiquement une traduction du poète latin Perse (Satire V); N. Boileau, Euvres poétiques, Paris, Firmin Didot frères, 1853, p. 194 ; URL : https://gallica.bnf.fr/ ark:/12148/bpt6k5442473z; consulté le 16.01.2019.

${ }^{27}$ Ibid., p. 34-35.

${ }^{28}$ Ibid., p. 35.

${ }^{29}$ Si psychologiquement l'homme est « sensation - sentiment - connaissance », philosophiquement, sa nature se définit par une aspiration incessante à la perfection, ce qui explique la notion du progrès (ou de la perfectibilité) de l'humanité, inconnue des Anciens, et formulée au XVIIe et au XVIII ${ }^{e}$ siècles, principalement en France (par Perrault, Fontenelle, Descartes, Turgot, Condorcet et SaintSimon); ibid., p. 111-121. 
le passage ", disait Montaigne $\left.{ }^{30}\right)$ : «Les plaisirs et les douleurs, les biens et les maux, n'ont aucune valeur absolue et constante $»^{31}$. Le bonheur de l'homme ne réside donc que dans son attitude envers la vie : « [...] tout notre bonheur consiste essentiellement et uniquement dans l'état avec lequel nous aspirons. / C'est ce que j'appellerais volontiers le ton de notre vie $»^{32}$.

«Aimer religieusement le monde et la vie » n'est possible que lorsque l'homme se défait de ses illusions concernant la vie future. Pas d'au-delà, pas de ciel ni d'enfer chez Leroux, à moins qu'on n'envisage le ciel comme synonyme de Dieu : il aurait alors un aspect incréé, virtuel et infini, et un aspect créé, manifesté, limité dans l'espace et dans le temps ${ }^{33}:$ «Le véritable ciel, c'est la vie, c'est la projection infinie de notre vie $\aleph^{34}$. La vie future ne consiste qu'en une renaissance sans fin de l'homme au sein de l'humanité ${ }^{35}$. Il n'y a pas de véritable amour pour l'homme en dehors de la nature et de la vie : c'est bien la raison pour laquelle le christianisme, à cause de son mépris du monde, n'a jamais pu vraiment réaliser l'amour de Dieu et du prochain, pourtant prêché par Jésus. Le regard fixé sur un ciel imaginaire détournait son attention de la « vie vivante $»^{36}$.

Il a fallu pour qu'on arrivât à ce profond dédain qu'on affecte aujourd'hui pour la nature et pour la vie, c'est-à-dire pour l'humanité, il a fallu, dis-je, pour arriver à se faire de la vie future les idées chimériques qu'on s'en fait, croire, comme en effet on l'a cru longtemps, que des révélateurs tombés de ce monde inconnu et tout à fait incorporel qu'on appelle le ciel étaient venus sur la terre. [...] Or, c'est parce que ce monde du ciel, cet empyrée des révélateurs et des saints, d'où descendait sur l'humanité vivante un terrible despotisme, n'existait réellement pas, que ce despotisme a pu être détruit à l'époque de la Réforme, et l'homme être émancipé de cette hiérarchie céleste imaginaire, qui engendrait sur la terre, sous le nom de Clergé, une hiérarchie correspondante ; c'est, dis-je, parce que tout ce ciel était chimérique qu'il a pu être renversé ${ }^{37}$.

La rupture métaphysique entre le monde et l'au-delà, principe même du contemptus mundi, n'a fait que générer l'égoïsme : d'une part celui, spirituel, de l'ascète et du dévot, d'autre part celui, hédoniste, du libertin incrédule ${ }^{38}$. Seule une réhabilitation de la nature et de la vie peut conduire à l'application réelle

\footnotetext{
${ }^{30}$ M. de Montaigne, Essais, éd. présentée, établie et annotée par P. Michel, t. 3, Paris, Gallimard, 1965, p. 44. Comme le montrera la conclusion, le nom de Montaigne n'apparaît pas ici par hasard.

${ }^{31}$ Ibid., p. 79.

32 Ibid., p. 78.

${ }^{33}$ Ibid., p. 184-188.

${ }^{34}$ Ibid., p. 182.

${ }^{35}$ Il s'agit-là de la thèse principale que Leroux défend dans son ouvrage. Il expose successivement à son appui les preuves par Virgile, Platon, Pythagore, Apollonius de Tyane, les sectes juives et Jésus-Christ.

${ }^{36}$ Ibid., p. 34.

${ }^{37}$ P. Leroux, De l'humanité, t. 2, op. cit., p. 416-418.

${ }^{38}$ Idem, De l'humanité t. 1, op. cit., p. 157-173.
} 
et socialement organisée du principe de l'amour. Égoïsme (amour de soi) et charité (amour de l'autre) ne feraient alors qu'un, car l'homme, conscient de son lien profond avec l'humanité, saurait bien qu'en aimant l'autre, il ne fait en réalité qu'aimer soi-même ${ }^{39}$.

On peut donc conclure qu'en réponse au contemptus mundi, Leroux propose une certaine philosophie de la vie, qui possède deux aspects essentiels. Premièrement, cette philosophie doit être située dans le contexte du développement à l'époque du romantisme de l'herméneutique philosophique ${ }^{40}$, dont les modalités de pensée se cristallisent au fur et à mesure que s'effrite la métaphysique traditionnelle. L'œuvre de Leroux est symptomatique à cet égard : critique radical de l'éclectisme philosophique d'inspiration hégélienne (représenté par Victor Cousin ou Théodore Jouffroy), il cherche à trouver un nouvel " axiome ontologique » qui servirait de point d'appui à la vie humaine :

On voit que par ce mot d'axiome ontologique j'entends quelque chose d'assez différent de cette science abstraite et tronquée qu'on appelle quelquefois dans les écoles actuelles ontologie. Il n'y a pas à faire de philosophie, si l'on ne brise à chaque instant les absurdes barrières que les psychologues modernes ont établies entre leurs élucubrations abstraites et la vie, c'est-à-dire la vie religieuse, morale et sociale à la fois ${ }^{41}$.

Il ne s'agit donc pas pour Leroux de chercher les solutions aux grands problèmes de l'homme dans une ontologie abstraite coupée de l'existence. Et même s'il paraît poser la question des fondements de l'être de façon assez traditionnelle, il le fait en réhabilitant et en affirmant le monde et la vie par rapport au principe métaphysique, et en réduisant de cette façon celui-ci à un horizon de la vie, à la fois présent dans le monde et inaccessible. Or, l'« axiome ontologique » dont il est question plus haut ne peut être fourni que par la religion - non pas l'ancienne religion instituée, mais une nouvelle religion qui se réfèrerait à l'expérience intégrale de l'humanité. Leroux constate donc qu'on ne saurait s'arrêter à l'œuvre de destruction menée par la philosophie des Lumières, mettant un terme aux formes religieuses idolâtres. Il nous invite en même temps à dépasser la rationalité stérile de la tradition philosophique, qu'elle mène au spiritualisme ou au matérialisme, et à repenser les traditions religieuses et leurs mythes, afin d'apprendre à " aimer religieusement le monde et la vie ». Il ne s'agit en effet pas de passer d'un extrême à l'autre et, ayant démystifié le mépris du monde, qui repose sur des chimères, commencer à aimer le monde de façon aveugle et confuse.

Deuxièmement, la philosophie de la vie proposée par Leroux possède un caractère éminemment pratique, qui la rapproche de la perspective métaphilosophique tracée

\footnotetext{
${ }^{39}$ Ibid., p. 173-175.

${ }^{40}$ G. Gusdorf, Les Origines de l'herméneutique, Payot, Paris 1988.

${ }^{41}$ P. Leroux, De l'humanité, op. cit., t. 1, p. XIV.
} 
par des chercheurs comme Pierre Hadot ou Juliusz Domański ${ }^{42}$. La philosophie n'est pas une question de spéculation, mais avant tout un mode de vie. Le fait que Leroux adopte une attitude critique par rapport aux écoles anciennes qui concevaient la philosophie de cette façon ne doit pas nous induire en erreur, car il voit aussi leurs mérites et leur rôle dans le progrès de l'humanité. Ce qui compte avant tout pour lui, c'est dépasser l'opposition fatale entre un idéalisme générateur du contemptus mundi (platonisme, stoïcisme, christianisme) et un matérialisme tout aussi faux et réductionniste (épicuréisme). Mais en même temps, il apprécie pleinement l'aspiration de ces anciennes écoles à répondre aux questions essentielles de la vie - à la question du souverain bien et du bonheur, en proposant un certain art de vivre. Le fait qu'elles se soient trompées dans leur quête de la sagesse ne signifie pas pour autant que cette quête en tant que telle est dépourvue de sens, bien au contraire. Leroux lui aussi donne sa réponse aux problèmes du bonheur et du souverain bien. Il place le premier dans une certaine attitude envers la vie (ce qu'il nomme le « ton de notre vie »), faite à la fois de détachement et d'engagement, une certaine façon d'aspirer, autrement dit de progresser, et le second, dans l'amour du monde et de la vie, qui consiste en une réinterprétation de la charité chrétienne prêchée par Jésus, mais délivrée du mépris qui pesait sur le monde. La « communion du genre humain, ou, en d'autres termes, la solidarité mutuelle des hommes » devient ainsi le « point fixe », l' « axiome ontologique $»^{43}$ qui brise l'opposition illusoire entre l'altruisme et l'égoïsme.

\section{Bibliographie}

Anthologie de Pierre Leroux, inventeur du socialisme, établie et présentée par Bruno Viard, Lormont, Éditions Le Bord de l'eau, 2007

Alexandrian, Sarane, Le Socialisme romantique, Paris, Éditions du Seuil, 1979

Bénichou, Paul, Le Temps des prophètes. Doctrines de l'âge romantique, Paris, Gallimard, 1977

${ }^{42}$ Voir à ce sujet: P. Hadot, Qu'est-ce que la philosophie antique?, Paris, Gallimard, 1995, surtout le chapitre «La philosophie comme mode de vie »; La Philosophie comme manière de vivre, entretiens avec Jeannie Carlier et Arnold Davidson, Paris, Éditions Albin Michel, 2001 ; J. Domański, La Philosophie, théorie ou manière de vivre? Les controverses de l'antiquité à la renaissance, préface de P. Hadot, Fribourg, Éditions universitaires Fribourg Suisse / Paris, Éditions du Cerf, 1996. La conception antique de philosophie comme mode de vie a été occultée par la venue du christianisme, qui a réservé l'aspect pratique à la réalisation de l'enseignement évangélique (notamment dans le monachisme), et qui a réduit la philosophie à une propédeutique théologique. La philosophie dès lors continuait son cheminement vers sa version purement spéculative et académique, admise dans le monde moderne (et rejetée par Leroux), tandis que la philosophie comme exercice spirituel et manière de vivre a été absorbée par la spiritualité chrétienne et incorporée à cette dernière (ce qui trouve son illustration exemplaire dans les Exercices spirituels d'Ignace de Loyola). On observe toutefois une certaine résurgence de la philosophie comme mode de vie à l'époque de la Renaissance, notamment chez Montaigne ou Érasme.

${ }^{43}$ P. Leroux, De l'humanité, t. 1, op. cit., p. XIV. 
Boileau Nicolas, CEuvres poétiques, Paris, Firmin Didot frères, 1853 ; URL : https:/gallica.bnf.fr/ ark:/12148/bpt6k5442473z; consulté le 16.01.2019

Bultot, Robert, " Mépris du monde, misère et dignité de l'homme, dans la pensée d'Innocent III ", Cahiers de civilisation médiévale, octobre-décembre 1961, $4^{e}$ année, $\mathrm{n}^{\circ} 16$, p. 441-456, URL : https://www.persee.fr/doc/ccmed_0007-9731_1961_num_4_16_1205; consulté le 10.01.2019 https://doi.org/10.3406/ccmed.1961.1205

Domański, Juliusz, La Philosophie, théorie ou manière de vivre? Les controverses de l'antiquité à la renaissance, préface de P. Hadot, Fribourg, Éditions universitaires Fribourg Suisse / Paris, Éditions du Cerf, 1996

Domański, Juliusz, $Z$ dawnych rozważań o marności i pogardzie świata oraz nędzy i godności człowieka, Warszawa, Wydawnictwo IfiS PAN, 1997

Evans, David Owen, Le Socialisme romantique : Pierre Leroux et ses contemporains, Paris, M. Rivière, 1948

Gusdorf, Georges, Les Origines de l'herméneutique, Payot, Paris 1988

Hadot, Pierre, Qu'est-ce que la philosophie antique?, Paris, Gallimard, 1995

Hadot, Pierre, La Philosophie comme manière de vivre, entretiens avec Jeannie Carlier et Arnold Davidson, Paris, Éditions Albin Michel, 2001

Houellebecq, Michel, H. P. Lovecraft. Contre le monde, contre la vie, Monaco / Paris, Éditions du Rocher, 1991

Leroux, Pierre, De l'humanité, de son principe et de son avenir, t. 1, Paris, Perrotin, 1845 ; URL : http://gallica.bnf.fr/ark:/12148/bpt6k454802b ; consulté le 10.01.2019

Montaigne, Michel de, Essais, édition présentée, établie et annotée par Pierre Michel, t. 3, Paris, Gallimard, 1965

Nietzsche, Friedrich, Ainsi parlait Zarathoustra, Paris, Éditions Gallimard, 1947

Viard, Bruno, Pierre Leroux, penseur de l'humanité, Cabris, Éditions Sulliver, 2009

Tomasz Szymański, titulaire d'un doctorat en sciences humaines (thèse : La théorie des correspondances dans l'œuvre de Charles Baudelaire, soutenue à Varsovie en 2009), actuellement en poste d'enseignant-chercheur à l'Institut d'Études Romanes de l'Université de Wroclaw (enseignant à partir de 2010). Champs de recherche et centres d'intérêt : littérature du XIX $\mathrm{X}^{\mathrm{e}}$ siècle (surtout romantisme) ; problématique philosophique (herméneutique, métaphilosophie, anthropologie) et religieuse (histoire des doctrines et des spiritualités, ésotérisme occidental, post-sécularité) ; histoire des idées (projet de recherche actuel : L'Idée de religion universelle en France au XIX ${ }^{\mathrm{e}}$ siècle).

\begin{tabular}{|c|c|}
\hline \multirow[t]{2}{*}{ cc creative } & $\begin{array}{l}\text { (C) by the author, licensee Łódź University - Łódź University Press, } \\
\text { Łódź, Poland. This article is an open access article distributed under } \\
\text { the terms and conditions of the Creative Commons Attribution license } \\
\text { CC-BY-NC-ND } 4.0 \text { (https://creativecommons.org/licenses/by-nc-nd/4.0/) }\end{array}$ \\
\hline & Received: 2019-01-30; Accepted: 2020-12-06 \\
\hline
\end{tabular}

